\title{
Trials at the ready: preparing for the next pandemic
}

In this Feature article by Ed Yong (BMJ 2012;344:e2982, doi:10. 1136/bmj.e2982) the job title for Mike Clarke, quoted initially in the third paragraph, should have been director of the Cite this as: BMJ 2012;344:e3240 All-Ireland Hub for Trials Methodology Research [not Director of the UK Cochrane Centre]. 